PPPL-3187 - Preprint: May 1996, UC-420, 426

\title{
A Procedure for Generating Quantitative 3-D Camera Views of Tokamak Divertors.
}

\author{
P. H. Edmonds, \\ Fusion Research Center, \\ University of Texas at Austin, \\ Austin Texas, 08543 \\ S. S. Medley, \\ PECPESO \\ HAV 281939 \\ OSTI \\ Princeton Plasma Physics Laboratory,
O. Box 451, Princeton, New Jersey, 0854
}

\begin{abstract}
A procedure is described for precision modeling of the views for imaging diagnostics monitoring tokamak internal components, particularly high heat flux divertor components. These models are required to enable predictions of resolution and viewing angle for the available viewing locations. Because of the oblique views expected for slot divertors, fully 3-D perspective imaging is required. A suite of matched 3-D CAD, graphics and animation applications are used to provide a fast and flexible technique for reproducing these views. An analytic calculation of the resolution and viewing incidence angle is developed to validate the results of the modeling procedures. The calculation is applicable to any viewed surface describable with a coordinate array. The Tokamak Physics Experiment (TPX) diagnostics [1] for infrared viewing are used as an example to demonstrate the implementation of the tools. For the TPX experiment the available locations are severely constrained by access limitations and the resulting images are marginal in both resolution and viewing incidence angle. Full coverage of the divertor is possible if an array of cameras is installed at 45 degree toroidal intervals. Two poloidal locations are required in order to view both the upper and lower divertors. The procedures described here provide a complete design tool for in-vessel viewing, both for camera location and for identification of viewed surfaces. Additionally these same tools can be used for the interpretation of the actual images obtained by the actual diagnostic.
\end{abstract}

\section{Introduction}

The very high heat loads on the TPX divertor targets require continuous temperature monitoring to anticipate and correct for any damaging fault conditions $[2,3]$. This monitoring will be done using infra-red cameras optically

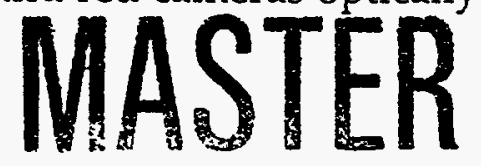


coupled to imaging elements located inside the vacuum system and viewing through penetrations in the plasma facing components.

An important requirement is that the cameras be optimally located in order to allow a complete view of the divertor structure, with sufficient resolution that the various fault conditions can be distinguished. Available camera locations are limited because of access restrictions and remote maintenance requirements. Viewing angles are limited by the passive stabilizer plates and other internal structures. It is desirable that the imaging systems be shielded from the full plasma radiated power to minimize first element coating and surface damage from heating and erosion.

A procedure for generating images showing both the available views for the various camera locations and for qualitatively estimating the appropriate viewing resolution and incidence angles is described using commonly available DOS based 3-D computer aided design, graphics and animation applications.

\section{Overview}

The paper is divided into four further sections. First, a general overview of the technique and a detailed description of a representative set of results is presented. Second, a calculation of the viewing performance, derived from the virtual camera views, is presented. Third, an analytic calculation of the image resolution and incidence angle is developed and the results compared with those obtained from the 3-D imaging technique. Fourth, a detailed description of the procedures used to generate the 3-D images is presented, to assist the reader in reproducing the results without excessive rediscovery.

The aim of the study is to provide a convenient technique for displaying camera views of in-vessel, particularly divertor, components and to indicate the expected resolution and viewing angles for the various surfaces. The procedure is to import a 3-D model of the relevant tokamak structures into a 3-D modeling application. In the application a "spotlight" is positioned at the camera location and this spotlight projects a pattern which exactly represents the camera field of view. Projections more or less perpendicular to the divertor surfaces are generated using viewing tools from the application. The views of these projected images then show the areas that are visible from the various camera locations and identify the achievable resolution and image quality. 
A simplified drawing of the TPX vacuum vessel, ports, limiter and divertor structures was made using AutoCad [4]. This drawing was used as the starting point for the study and is shown in Fig. 1. Included in this drawing is the nomenclature used for the various components.

The divertor and limiter outlines were lofted into 3-D shapes. The drawing is shown in Fig. 2 and includes the poloidal location of the imaging optics used in this study.

The drawing was imported into the graphics and animation application, 3-D Studio [5]. This application is primarily used as an artist's tool and includes detailed lighting capability. The procedure used in this study is to represent the imaging systems in the experiment with spotlights which project a checkerboard pattern. A typical view, from the spotlight location, of this checkerboard pattern is shown in Fig. 3.

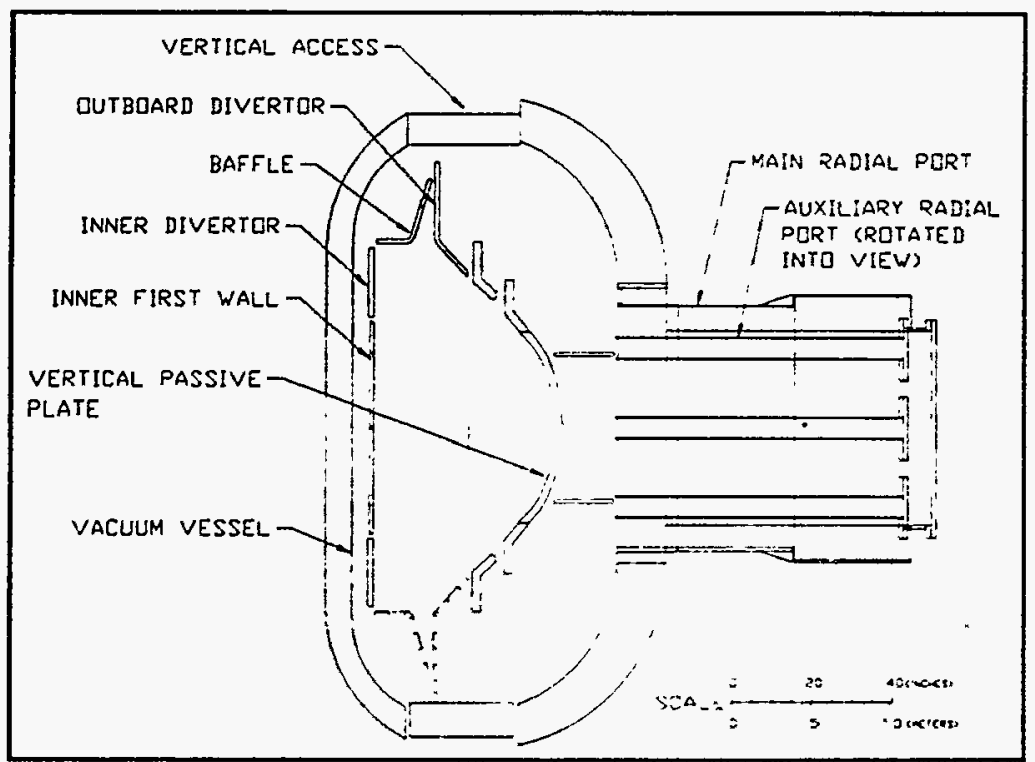

Fig. 1. Elevation view of TPX core including vacuum vessel and divertor/first wall outlines. Auxiliary port is rotated into view. 


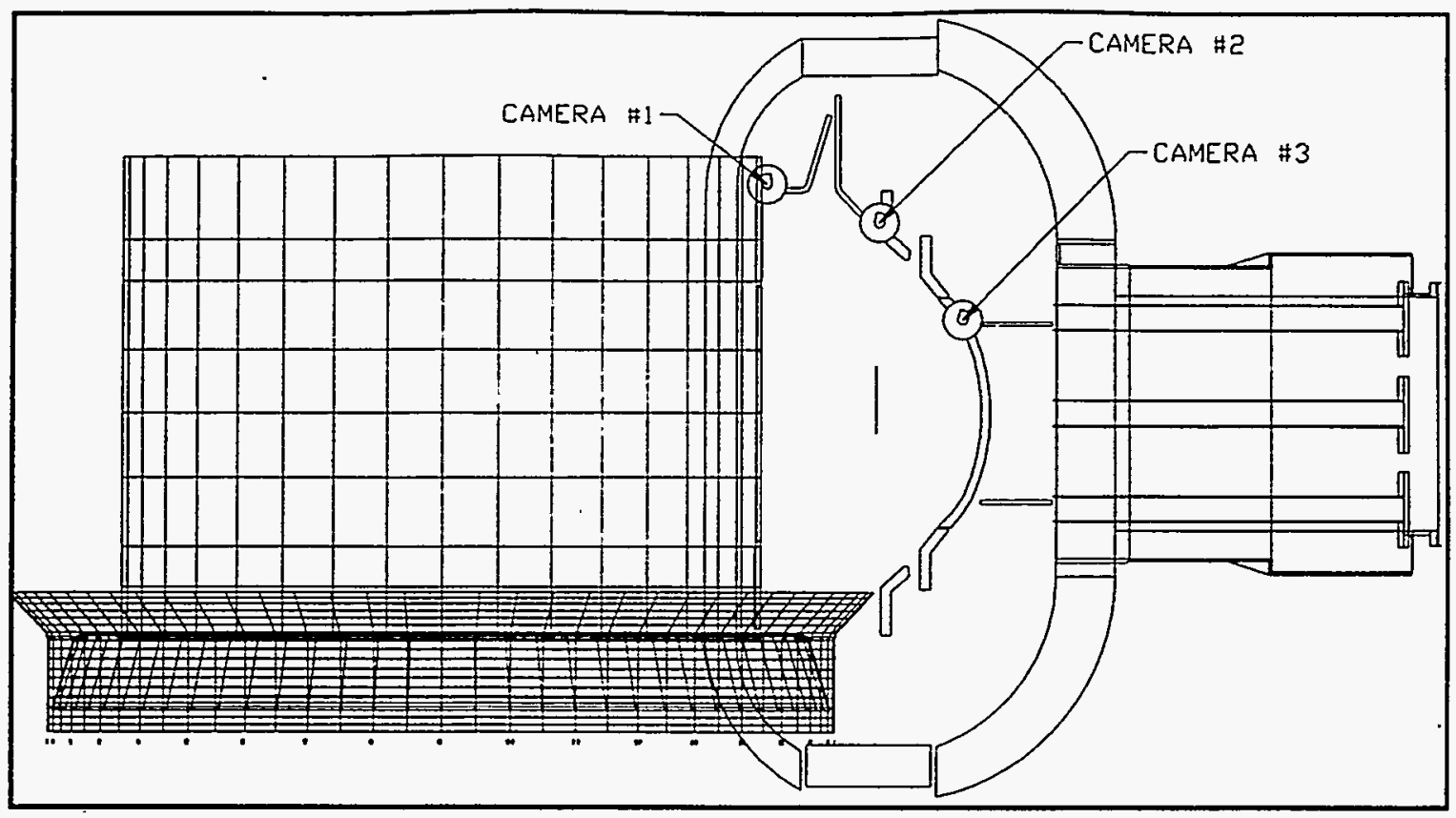

Fig. 2. 3-D developed drawing showing location of IR cameras (spotlights in application) prior to loading into 3-D Studio

In the actual experiment, if a camera was located at the same place as the spotlight in the model, there is an exact correlation between the location, shape and size of the pattern and the camera image, assuming that the field of view is

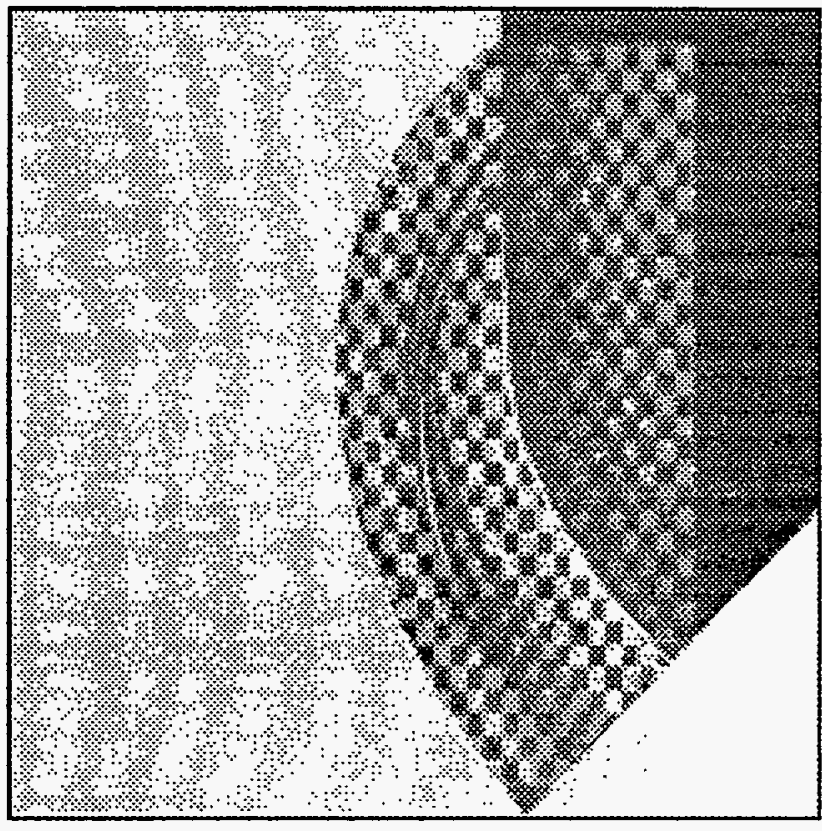

Fig. 3 View of spotlight \#2 (IR camera \#2) showing projector image of 32 by 32 square checkerboard. the same in both cases. For instance, if the pattern is 32 squares on a side and the camera diode array is 512 pixels square, then each pattern square corresponds to 16 pixels.

The images in 3-D Studio are obtained by appropriately generating a view, defined in the application by a "camera" and then rendering the resulting image. The images correctly indicate both perspective and shadows and the projected image of the spotlight represents the image that would be viewed by a real camera in the experiment. To indicate expected viewing areas and resolutions, the 3-D Studio "camera" was placed so as to view normal to the relevant divertor surface. Scale was indicated in the original 3-D model 
by appropriate markers. In order to view the inner divertor the "camera" needs to be located outside the divertor structure and has to see through the outboard divertor. Such magic is possible with 3-D Studio. A surface can be constructed that is transparent to viewing but projects shadows. Multiple spotlights can be installed so that the projected checkerboard patterns represent the areas visible for cameras, in the real experiment, located at the corresponding positions. In addition, spotlights can be added at different toroidal locations to indicate the extent of toroidal coverage.

The critical resolution views are of the cylindrical outboard divertor plate and the inner baffle cone. The measurements, results and discussions presented in this paper concentrate on these views. The work can be trivially extended to include other views.

As an example, Fig. 4 shows the rendered image obtained with spotlight No. 2 located at the $180^{\circ}$ toroidal position (see Fig. 2). The checkerboard pattern is 32 squares on a side. The 3-D Studio "camera" is located at the same vertical elevation as the divertor and the "camera" view is from inside, radially out. The "camera" location is shown in Fig. 13 and Fig. 14 as camera No. 1. These figures are taken from the 3-D Studio computer display.

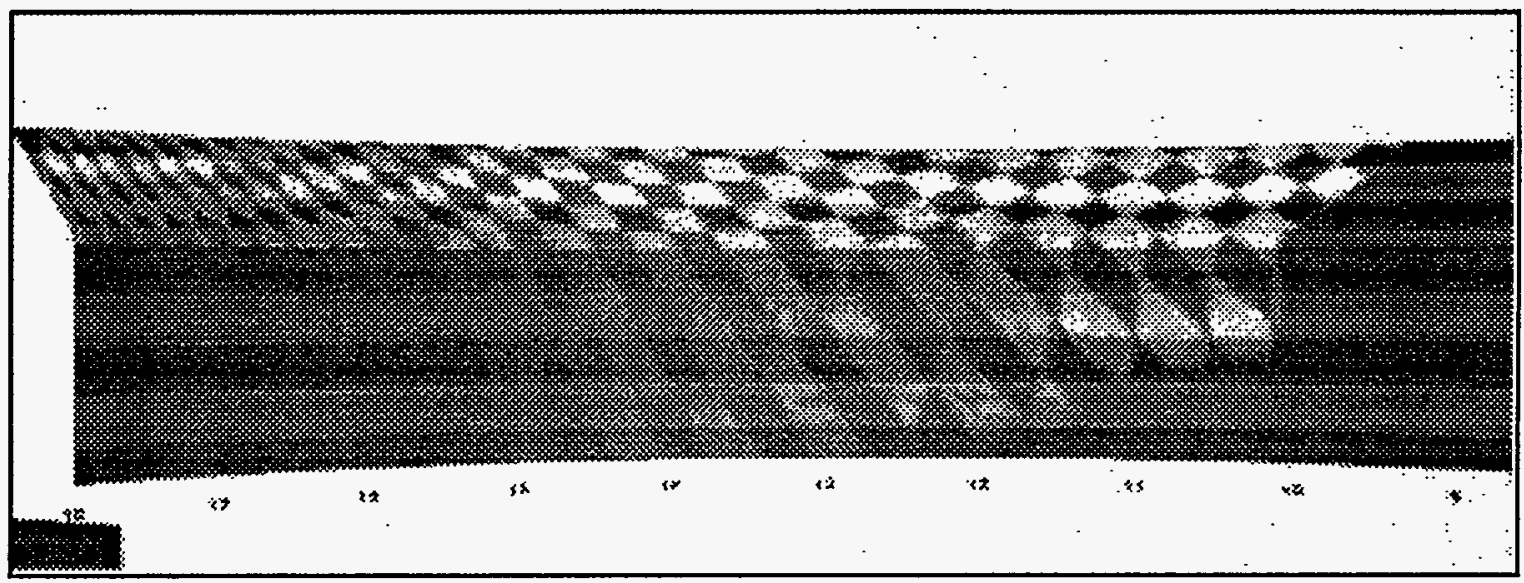

Fig. 4. View of outboard divertor with spotlight No. 2 turned on.

The image projected onto the outboard divertor cylindrical plate is limited to a toroidal angle of less than between $150^{\circ}$ and $140^{\circ}$ by the self shadowing and tangency of the divertor viewing line. The view from about $120^{\circ}$ to $100^{\circ}$ is gradually shadowed by the inner baffle and is cut off at about $100^{\circ}$ by the inner divertor and limiter shadow. Other cases will be described in Sect. 5 .

An estimate of the resolution can be made by comparing the size of the checkerboard pattern to the alternating horizontal bands (these are four inches apart) and of the approximate viewing angle by estimating the aspect ratio of the checkerboard pattern. For an aspect ratio of unity the view is normal.

The information required for most design activities, such as camera location and orientation, visible area and expected image quality, will be available from the results described to this point. 
3. Measurement of image resolution and viewing incidence angle.

In order to further quantify the image resolution a rectangular panoramic image was constructed from a mosaic made up of multiple images with the "camera" pivoted at the tokamak axis. A difficulty arises if the line of sight is along a diagonal; the checkerboard pattern is not necessarily aligned along this sight line and the ratio of the two sides will not reflect the viewing angle. For this more quantitative part of the study the square checkerboard projector pattern was replaced with an array, 64 on a side, of annuli. This panoramic view, for the same spotlight and "camera" positions as Fig. 2, is shown in Fig. 5.

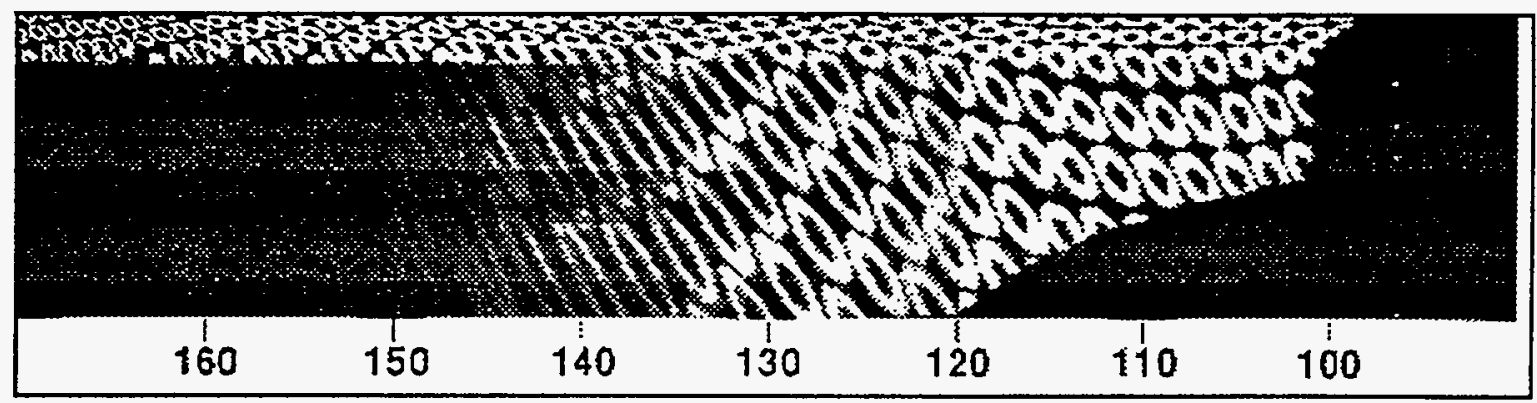

Fig. 5. Mosaic reconstruction of panoramic view of outer limiter with spotlight No. 2 and 64 element annular projector image.

For analysis, the length of the long and short axis of each pattern were measured and, assuming the individual patterns to be ellipses, the individual pattern centers, axis lengths and aspect ratios calculated.

Taking the length of the long axis of each ellipse, $\lambda$, as the appropriate dimension, the resolution, $\rho$, is defined as

$$
\rho=\lambda \times \frac{N_{\text {canck }}}{N_{\text {pual }}} \quad \text { where } N_{\text {check }} \text { is the number of patterns on one }
$$

side of the checkerboard, $N_{\text {pixel }}$ is the number of pixels along one side of the camera imaging array and the field of view of the projected pattern matches that of the camera. (Note that the projector pattern is surrounded by a mask and the effective field of view of the projected image is smaller than for the spotlight.)

The angle of incidence is defined as $\theta$, where

$\cos \theta=\eta \quad$ and $\eta$ is the aspect ratio of the ellipse, always taking the value less than unity. For normal incidence $\theta=0^{\circ}$ and for tangential incidence $\theta=90^{\circ}$. Contour plots of the resolution and incidence angle are shown in Fig. 6 and Fig. 7. Units of resolution are inches.

For much of the toroidal range of view only one or two annuli are complete and only a single data point is available. For these locations the contour lines are clipped short and to not necessarily indicate a direction. The accuracy is estimated at about $25 \%$. The bold outline represents the boundary of the projected pattern. This boundary extends from about $100^{\circ}$ to $145^{\circ}$, although the bottom of the view is clipped between $100^{\circ}$ and $120^{\circ}$. 


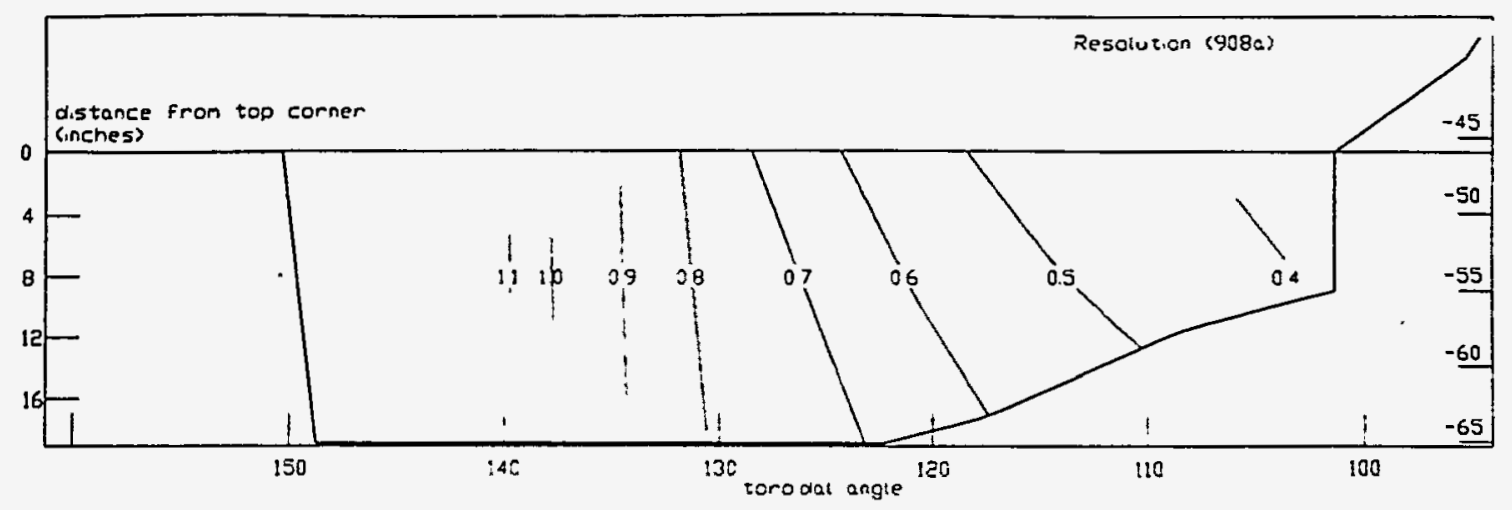

Fig. 6. Contour plot of resolution for single spotlight, No. 2, illuminating the outside limiter. Resolution units are inches, assuming the camera has 512 by 512 pixels.

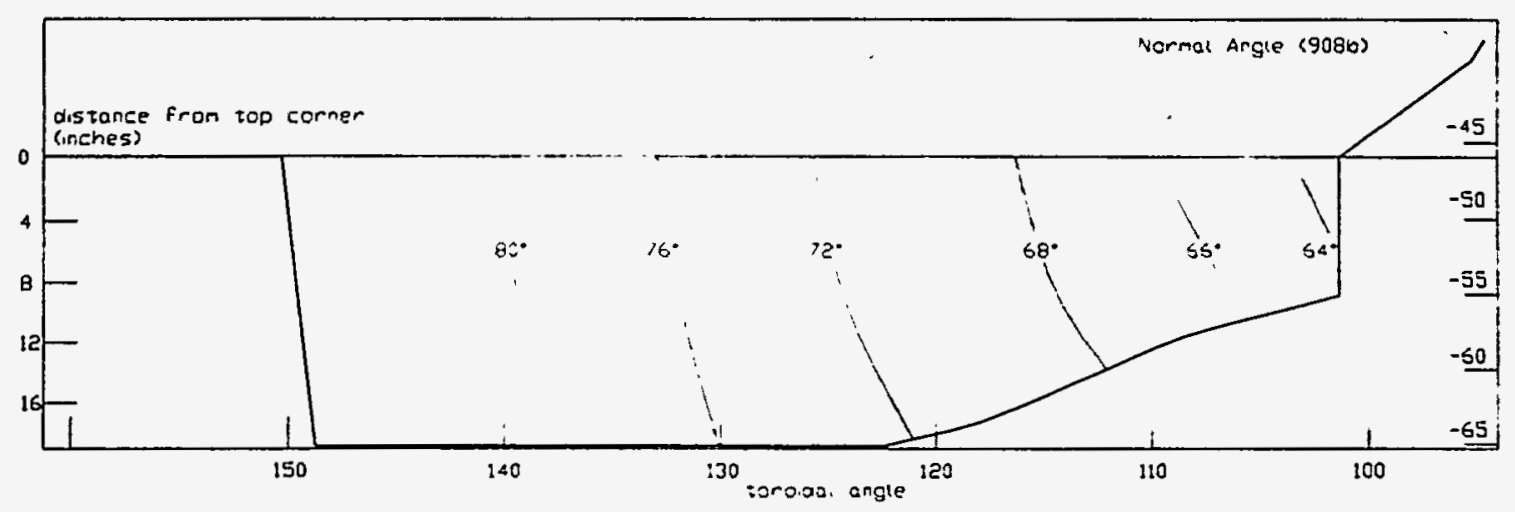

Fig. 7. Contour plot of incidence angle for single spotlight, No. 2, illuminating outside limiter.

For eight cameras installed at $45^{\circ}$ intervals, the toroidal coverage would be essentially $100 \%$. If each camera views both clockwise and counter-clockwise, the fields of view overlap as mirror images. This can be seen from Table I, which shows the fields of view for this case: cameras $90^{\circ}$ apart viewing both ways and poloidally located at position No. 2. However, for much of the view the incidence angle is greater than $70^{\circ}$ and the resolution worse than 1 inch, so for this case the quality of the imaging is marginal. 
Table I Approximate viewing range for cameras located at $45^{\circ}$ toroidal intervals and poloidal location No. 2.

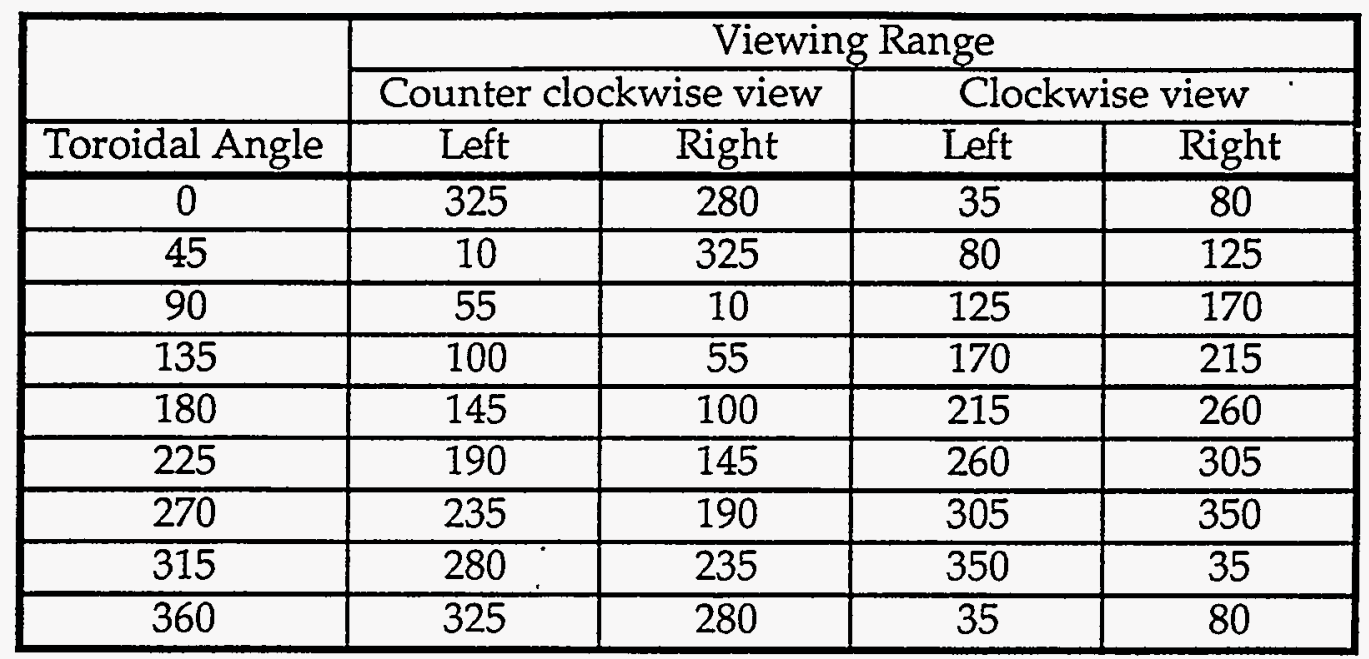

For views with a camera located at position No. 1, the resolution and coverage of the outer limiter is improved but all views of the inner baffle are essentially tangential. For views with a camera located at position No. 3, essentially the same result as position No. 2 is obtained. The toroidal view is somewhat improved as the camera is able to view further past the center column, but the resolution and incidence angles are somewhat worse. Images of these cases are included in the detailed description section of this paper.

\section{Analytic calculation of resolution}

In order to validate the resolution and viewing incidence angle results, an analytical calculation of the image quality was developed. The calculation projects the divertor surface coordinates onto the imaging plane and is applicable to any case where an array describing the divertor surface can be constructed. The calculation is applied to both the case described in Sect. 3 and also for a viewing system or camera located at position No. 3, a more desirable experimental location.

Consider the geometry shown in Fig. 8. If the angle between the $r$ pixel sight line (coordinates $u, v$ on the image plane, viewing the point $n$ on the divertor surface) and the camera sight line is $\phi$, then for an elemental angle about this sight line, $\delta \phi$, the ellipse projected onto the image plane has a long axis of length given by

$$
a=F \times \delta \phi \times \sqrt{\frac{1+\tan ^{2} \gamma \cos ^{2} \phi}{1+\tan ^{2} \gamma}}
$$

where $F$ is the distance from the camera lens to the image plane and $\gamma$ is the angle between the surface normal at the divertor $(n p)$ and the line from the camera axis to the pixel $r$, (or) projected along the camera sight line (op). 
In order to avoid the complication of calculating the angle $\gamma$, which requires a description of the camera orientation, the radical will be ignored. This at most underestimates the resolution for a corner pixel by about $10 \%$ for the camera field of view, $\psi$, of $42^{\circ}$ described here. Similarly, if the angle between the sight line and the normal to the divertor viewing point is $\theta$, the larger axis of the ellipse projected onto the divertor surface by this elemental angle is $b=L \times \delta \phi / \cos (\theta)$, where $L$ is the distance from the camera point to the viewing point on the divertor $(n)$.

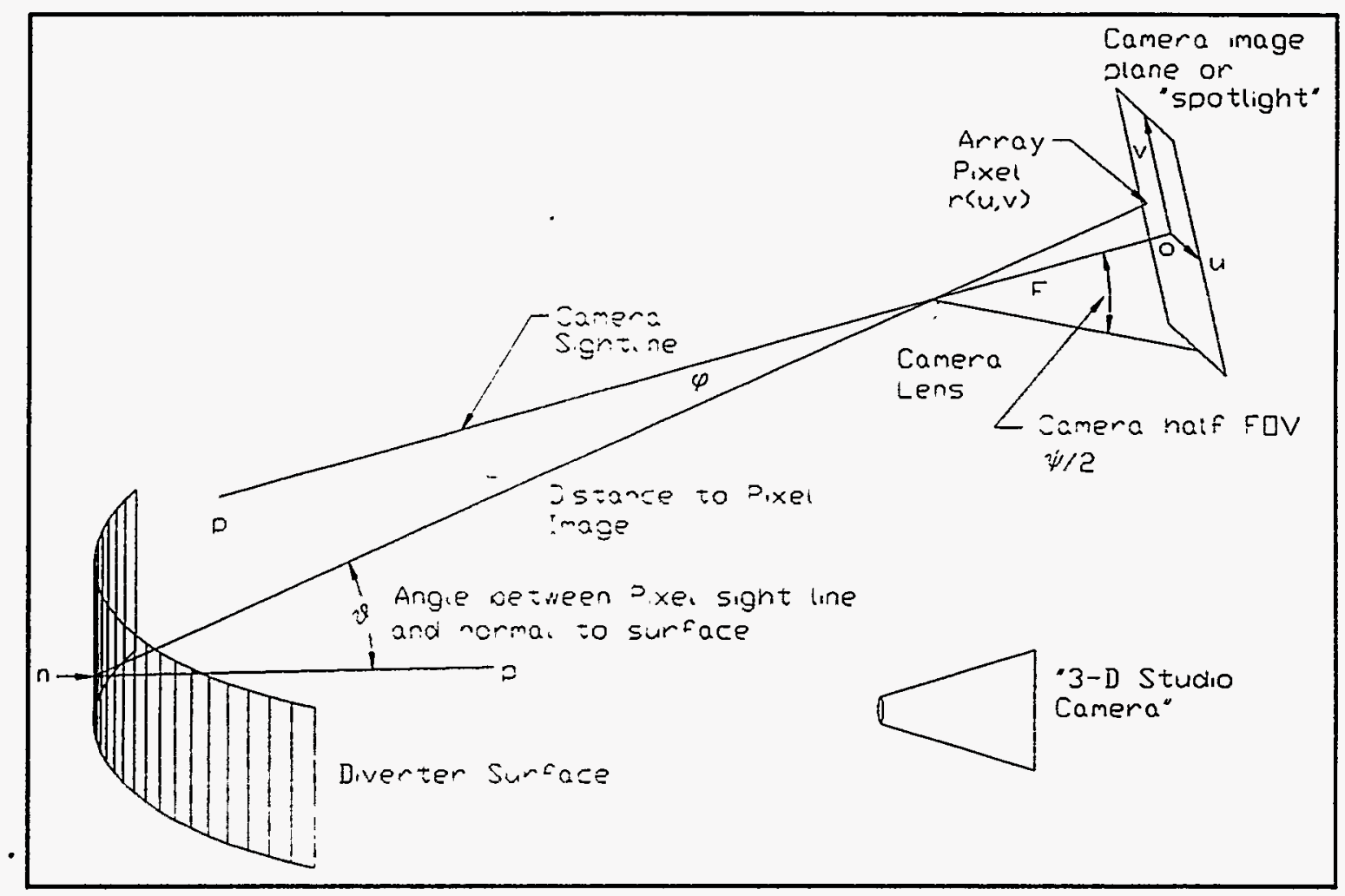

Fig. 8. Geometry details for calculation of image resolution and viewing angle.

Then the ratio of the image to pixel size, $b / a=L /(F \times \cos \theta)$.

Assume a square image array $2 \times N$ pixels on a side. Then the length of a single pixel is $I_{p}=F \times \tan (\psi / 2) / N$.

And finally the resolution, $\rho$, which is defined as the image of a single pixel projected onto the divertor

$$
\rho=\frac{L \times \tan (\psi / 2)}{N \times \cos \theta}
$$

A program was written to calculate the values of $\rho, \theta$ and $\phi$ for an equally spaced grid on the surface of the outer divertor for the same conditions used for the resolution calculation described in Sect. 2 (Appendix II). The analytic calculation gives no information on the viewed image and the array limits were taken from Fig. 5. 
The results of the calculation are shown in the contour plots, Fig. 9 for the resolution and Fig. 10 for the viewing angle. The contours are essentially straight or slowly curving lines. The structure or meanders are a consequence of the spline fits used by the contour generating program and should be disregarded. These figures should be compared with Fig. 6 and Fig. 7 respectively. For most of the range the agreement is excellent for both the resolution and for the angle of incidence.

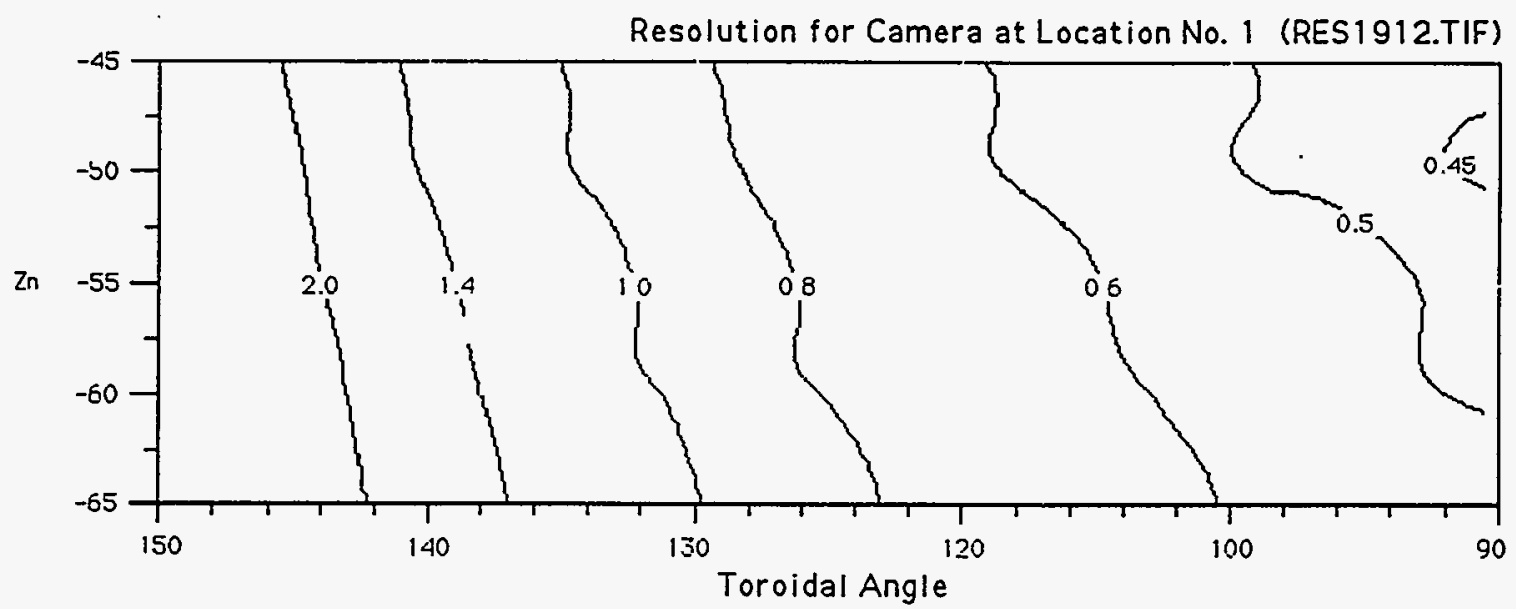

Fig. 9 Contour plot of resolution for camera located at position No. 1. Camera field of view is $42^{\circ}$ and array is 512 pixel square.

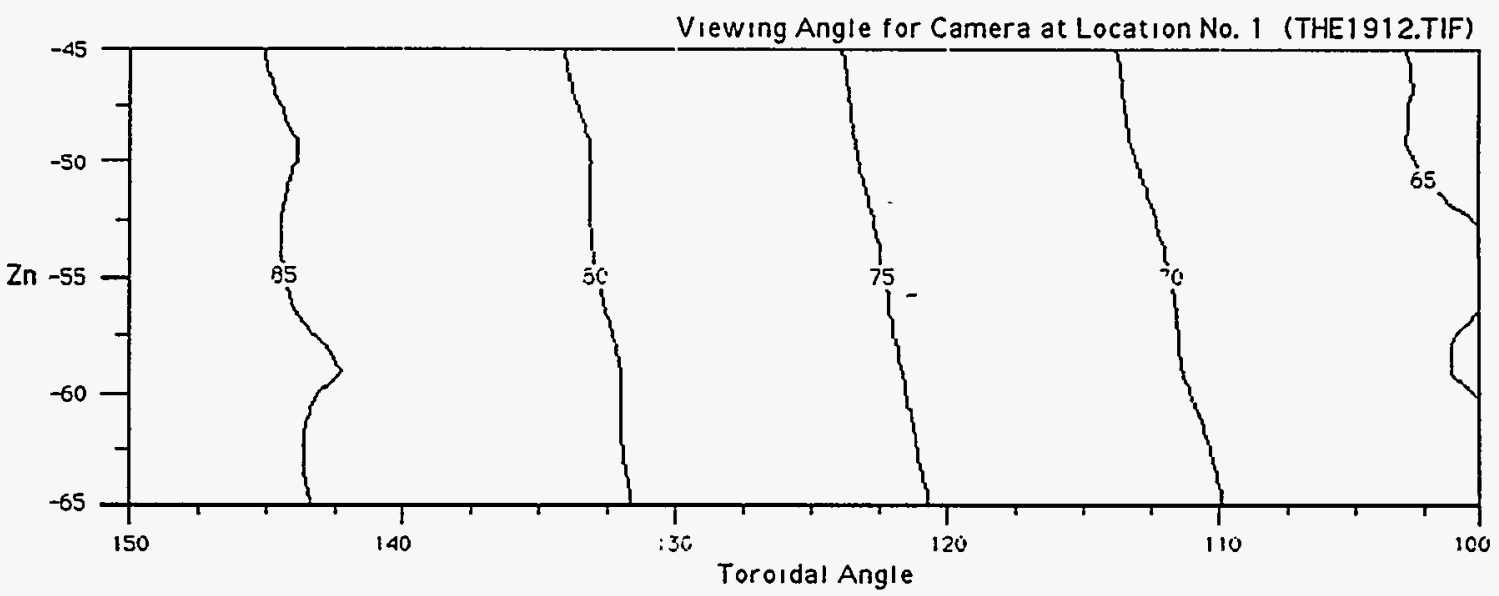

Fig. 10. Contour plot of viewing incidence angle for camera located at position No. 1. Camera field of view is $42^{\circ}$ and array is 512 pixel square.

The calculation was repeated for the camera location No. 3. The results are shown in Fig. 11 for the resolution and in Fig. 12 for the viewing incidence angle. The corresponding rendered image is shown in Fig. 18, note that this figure includes two projected images. 


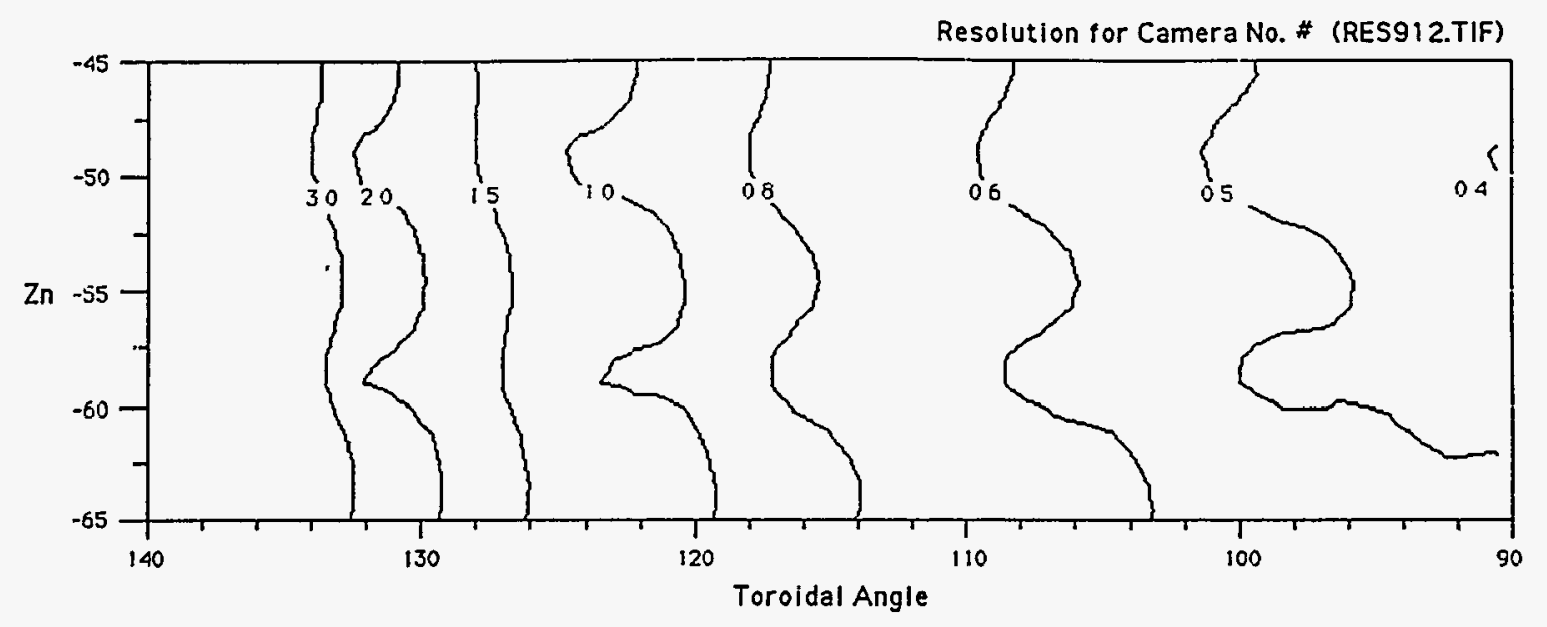

Fig. 11. Contour plot of resolution for a camera located at position No. 3. Camera field of view is $42^{\circ}$ and image array is 512 pixel square.

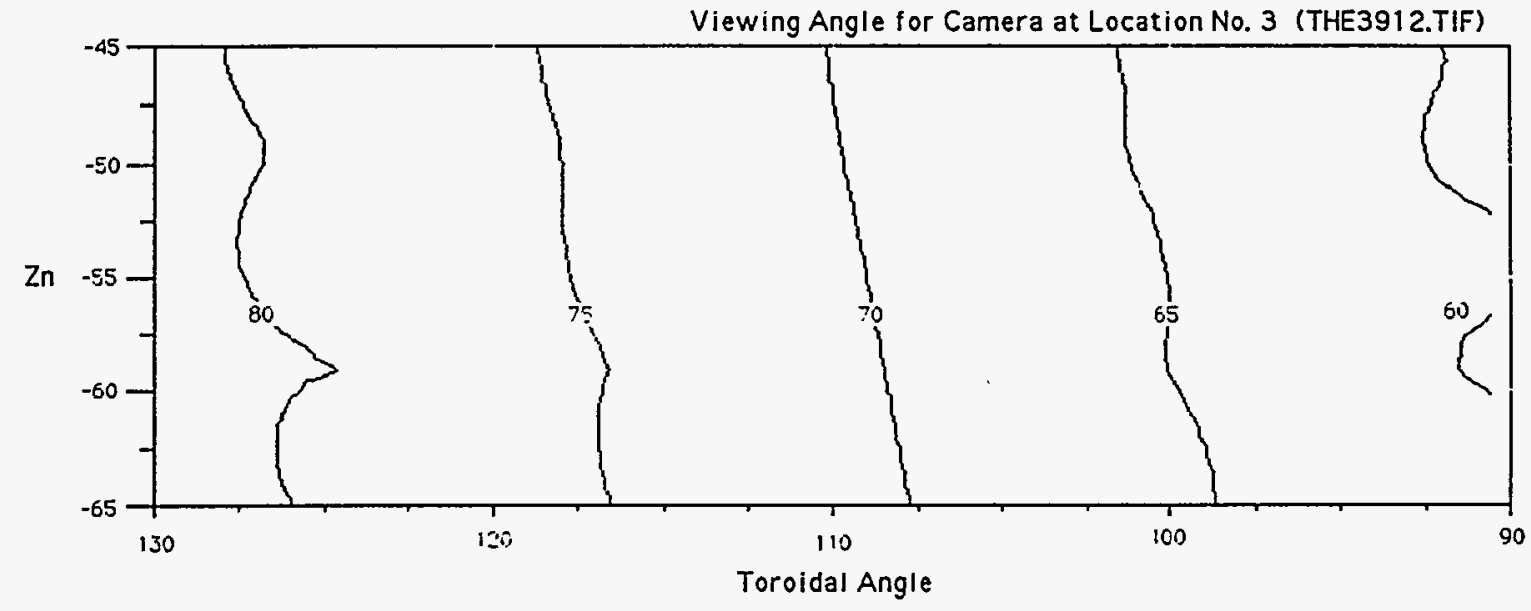

Fig. 12. Contour plot of viewing incidence angle for a camera located at position No. 3. Camera field of view is $42^{\circ}$ and array is 512 pixel square.

The procedure described above can readily be extended to include the resolution expected for any surface geometry. This calculation combined with the ability of the 3-D visualization procedure to indicate the range of view provides a complete solution to the camera view problem.

\section{Description of technique and procedures}

5.1. Dimensions, drawing sources and applications.

The divertor and baffle dimensions are taken from the TPX Engineering Change Proposal No. 0006 Rev. 1 (ECP 6). The location of the outer passive stabilizer plates or kink cage is taken from the TPX drawing \# 9411311B.DRW (5-05-95). The overall cross-section view of the TPX vessel and access locations was assembled from TPX drawings Nos. 9412101.SH2.P4 and 9412101.SH2.P5. This does not affect the procedures described here. The results presented here are for reference only and are provided in order to demonstrate the technique. 
The actual camera locations and sight lines will be provided by the diagnostics subcontractor and are expected to vary from the locations assumed here.

The primary software programs and applications used were AutoCad [4] and 3-D Studio [5]. 3-D Studio is a three dimensional modeling and animation application and consists of a coupled suite of modules, including a 2-D drawing module, 2-D Shaper, a conversion to 3-D module, 3-D Lofter, the main 3-D module, 3-D Editor, an animation module, Keyframer and a materials editor. All these programs are DOS based and were run on a $33 \mathrm{MHz}$ Intel 486 with $32 \mathrm{MB}$ of RAM. The screen dumps were converted to monochrome images using HiJaaK [6]. The contour plots were generated using DOS based graphing and plotting application PSPLOT [7]. The excellent MAC shareware application GraphicConvertor [8] was used to assemble the mosaic reconstruction for the panoramic view, as a general graphics viewer and conversion application and for editing and labels for the contour plots.

\subsection{Drawing development}

The first step was to import the TPX drawing files from the TPX file server into AutoCad, using the IGES ASCII file format. The resulting files were excessively large (of order tens of megabytes) and were overdrawn using AutoCad commands (overlay was ensured by only using "osnap" settings, as "end", "center", etc.). The outboard divertor and inner baffle were directly drawn using the dimensions from the ECP 6 document. The 2-D AutoCad drawing used for this study is shown in Fig. 1, which includes the nomenclature for the various parts.

A problem with 3-D images is the identification of scale. In order to allow quantitative measurements, the single curvature (straight lines in 2-D) components of the divertor baffles were divided into sections four inches long. These sections were alternately surfaced with white and gray material in the final model. In anticipation, each section was given a unique layer, lettered consecutively from one end of the baffle to the other (as IN1, IN2, etc.) in order to simplify the identification following import into 3-D Studio.

The drawing was then prepared for import into 3-D Studio. Experience indicates that as many operations as possible, and in particular, all lofting (conversion to 3-D) commands should be carried out in AutoCad prior to import into 3-D Studio. The lofting procedures available in 3-D studio are not particularly suitable for the precision required for engineering studies (3-D Studio is primarily an artist's tool and in general objects are located by eye rather than by direct coordinate entry). All the elements of the drawing required in 2-D outline (vessel, ports, passive stabilizer, etc.) were converted into "polylines" or 3-D surfaces. Any elements not converted into closed shapes are ignored by the conversion routines.

The outboard divertor and inner baffle elements were lofted to 3-D meshes using the "revsurf" command. The m-mesh value, "tabsurf1", was set to 36 and the finish angle at $180^{\circ}$; the increment is then $5^{\circ}$. The $n$-mesh value, 
"tabsurf2", was set to 2, which is the lowest permitted value. Each 4 inch element (a separate layer) of the outboard divertor and the inner divertor baffle was separately colored and lofted. The inner divertor and inner first wall were converted to 3-D meshes using a "tabsurf1" setting of 18; the corresponding toroidal resolution is $10^{\circ}$.

A set of digits was drawn in the 3-D Studio module 2-D Shaper using the lowest step size that produced recognizable numbers. The file was saved in $d x f$ format and imported into the AutoCad drawing. These digits were then inserted at the appropriate angular settings below the baffle gap. The procedure used was to assemble the two digit numbers at the zero angle and radius location of the baffle slot and then to rotate them through the required angle. Two perpendicular views in "vports" are required to make the "move" and "rotate" operation. The UCS has to be switched between the two views for each command. This awkward procedure was necessary because the AutoCad numbers are shape files and can not be converted into 3-D Editor objects.

The completed 3-D developed drawing including the location of the IR cameras (spotlights in the 3-D Studio application) used for this study is shown in Fig. 2. Studio.

The drawing was then saved as a $d x f$ file ready for importing into 3-D

\subsection{General Procedures}

The aim of the study is to generate projections more or less perpendicular to the divertor surfaces showing the areas that are visible for the various camera views and identifying the achievable resolution. This is done by replacing the imaging objects, IR cameras in the actual experiment, with 3-D Studio "spotlights". Each spotlight projects a checkerboard pattern. For the screen display the spotlights used different colors to highlight the overlap of the individual projector ( $\mathbb{R}$ camera) positions.

The 3-D studio "cameras" were located to generate approximately normal views of the divertor or baffle. Except for the panoramic views, to be discussed later, the "camera" was located fairly distant from the camera target in order to reduce the perspective distortion. The "camera" position and field of view and the "camera" target position were then adjusted so that the "camera" view included all the surfaces illuminated by the spotlights. This "camera" frame can be viewed by including the appropriate camera in the screen display using the menu command "views-viewports" and selecting one of the viewports as a camera view. The view was then rendered and saved as a 16 bit grayscale tif file.

The outline of the projected checkerboard pattern indicates the areas that can be viewed with the IR camera and the size of the projected checkerboard indicates the reciprocal resolution (a large checkerboard pattern represents low resolution). It is important that the checkerboard projection extents exactly corresponds to the simulated camera view. This can be demonstrated by rendering the relevant spotlight view. The rendered view for spotlight No. 2 (in the actual experiment this is the IR camera view) is shown in Fig. 2. An undocumented feature, discussed in detail later, is that the extent of the projected 
image is somewhat smaller than the extent of the spotlight without a projector, the projected pattern is surrounded by a mask.

Viewing the baffle from outside requires looking through the outboard divertor (and visa versa). A feature of 3-D studio is that objects may be constructed such that although they cast shadows they are themselves invisible. This is achieved by using one sided materials only and setting the normals of the outboard divertor facing inward and of the inner baffle facing outward. Reversed normals are a frequent cause of undesired objects appearing in the rendered images. The "render-setup-shadows" was set to "bitmap". The setting "ray trace" was used for the qualitative study in Sect. 3 in order to produce a sharper image, at the expense of a longer render time.

\subsection{Spotlight projector screen bitmaps}

The checkerboard bitmaps used for the projector screen were custom made for the study. First a simple square "quad" was created in the 2-D Shaper program and "grid" and "snap" were enabled. This object was imported into the 3-D Editor using the "create-object-get shape" command with "grid" and "snap" enabled at the same spacing as for the 2-D Shaper. The object was assigned "matte white" material. A succession of "create-object-copy" followed by "createobject-attach" commands were used to create the checkerboard. For most of the studies a 32 cell square checkerboard was used. A higher resolution 64 cells on a side and a 64 cells on a side array of annuli was constructed for the quantitative study. A gif bitmap file of the checkerboard was generated using the "renderobject" command in the "Top ( $(X / Z)$ " viewport. This gif file was loaded into each spotlight using the menu "projector" command.

The projected image from the spotlight is surrounded by a frame and the angular size of the spotlight "hotspot" is not the same as the angular spread of the projected pattern. The effective camera field of view was obtained by projecting the spotlight onto a grid at a selected distance and measuring the size of the pattern. This measured field of view was used in the calculation in Sect. 4.

\subsection{3-D Studio details}

The $d x f$ file drawing was loaded into 3-D studio using the "derive objects from layer" selection. Using the materials editor, a white and a gray single sided material was defined for the alternating outboard divertor and inner baffle layers. This is most easily done using the "select-object-name" command and selecting either the odd or even object name. This requires that the objects be on separate, consecutively numbered layers in the AutoCad drawing.

The outboard divertor objects were selected and the direction of the normals reversed to point inwards using the "surface-normals object flip" command. This is an awkward move as it is difficult to identify the normals directions. If they are set incorrectly, unpredictable results are obtained. It is suggested that the file be saved prior to this step and if any unexpected event occurs then reset and reload the file.

Three projector lights were added, in the plane of the vacuum vessel outline, at the camera locations shown in Fig. 2. 
Table II. Coordinates of Spotlights/IR Cameras

\begin{tabular}{|c|c|c|c|c|c|c|}
\hline Spotlight \# & \multicolumn{3}{|c|}{ Spotlight Location } & \multicolumn{3}{c|}{ Target Location } \\
\hline & $\mathrm{X}$ & $\mathrm{Y}$ & $\mathrm{Z}$ & $\mathrm{X}$ & $\mathrm{Y}$ & $\mathrm{Z}$ \\
\hline 1 & 66.0 & 47.0 & 0.0 & 69.3 & -39.8 & -32 \\
\hline 2 & 89.0 & 39.0 & 0.0 & 66.4 & -40.2 & -41.6 \\
\hline 3 & 106.0 & 19.0 & 0.0 & 47.0 & -46.0 & -28.0 \\
\hline 4 & 0.0 & 39.0 & -89 & 41.6 & -40.2 & -66.4 \\
\hline 5 & 0.0 & 19.0 & -106.0 & 28.0 & -46.0 & -47.0 \\
\hline
\end{tabular}

In general, objects are positioned by eye using the mouse and accurate location requires a high resolution "snap" setting and zoomed views. However, for some 3-D Studio operations the coordinates can be directly entered using the. keyboard. Fortunately keyboard entry can be used for lights, and they, or the equivalent IR camera, can be exactly located. The command requires the entry of the target location as well. Initially this can be entered as a dummy number, $0,0,0$, for instance, the target can then be positioned using the "lights-spot-move" command.

The checkerboard bitmap

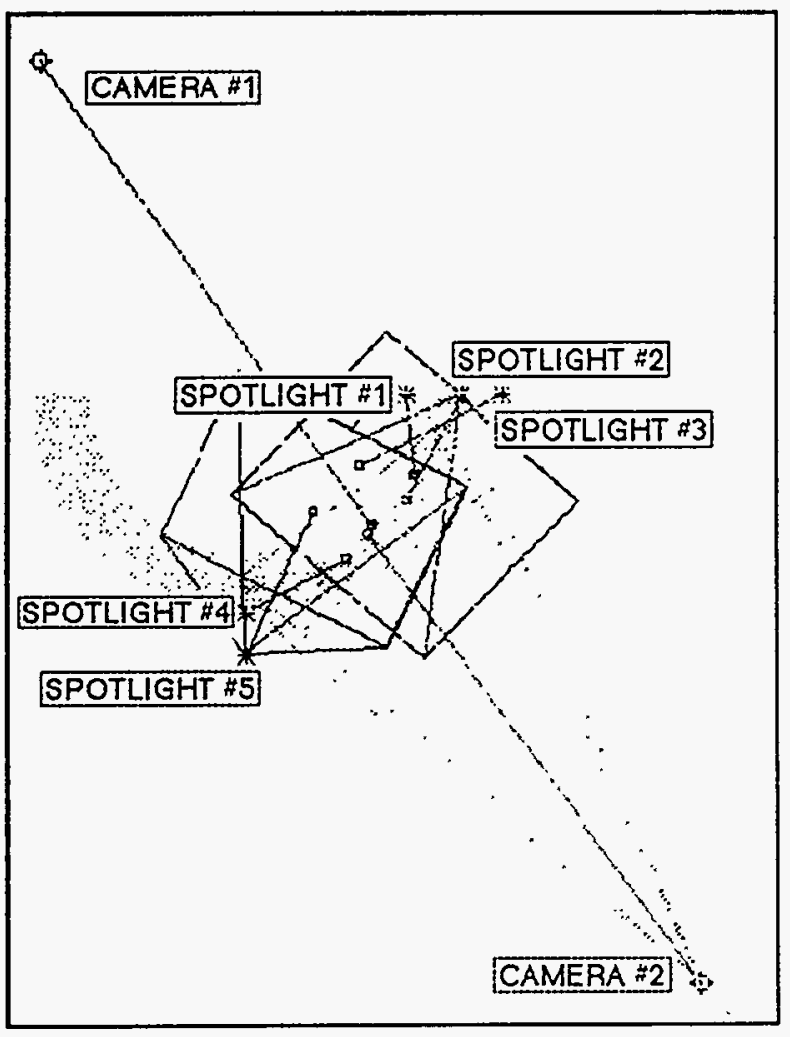

Fig. 13. Screen dump of plan view $(X / Z)$ of 3-D Editor display showing location of "spotlights" and "cameras". was used as the projector slide. As mentioned earlier, care must be taken to correctly define the field of view, allowing for the virtual mask surrounding the projector slide. The spotlights were differently colored so that each projected image could be identified in the screen view. Two additional lights were located at the same poloidal locations but 90 toroidal degrees away, looking in the counter direction, in order to represent cameras located at the next available viewing station. The coordinates for these two spotlights were derived from spotlights No. 2 and No. 3 in order to properly define the views. The coordinates used for the spotlights/IR cameras are given in Table II.

Two "cameras" were located so as to generate near perpendicular views of the inner baffle and outboard divertor. The 


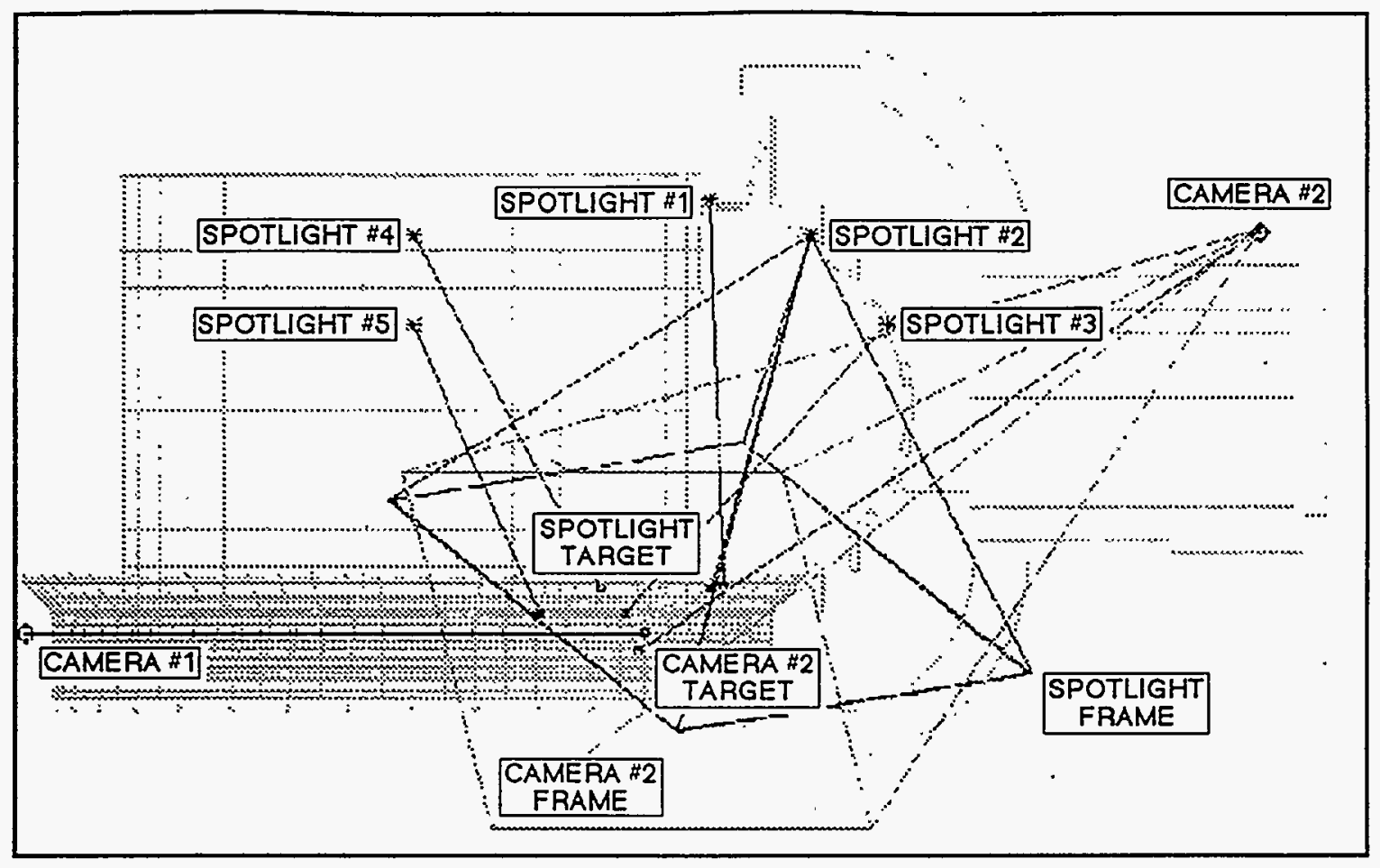

Fig. 14. Screen dump of elevation view $(X / Y)$ of 3-D Editor display showing location of "spotlights" and "cameras".

camera distance and field of view were adjusted to image the divertor over a $90^{\circ}$ toroidal range.

To provide a background light level and to prevent large areas of black, a low level ambient light was also included.

The location of the lights and the cameras can be seen in the screen views shown in Fig. 13 and Fig. 14, the spotlight No. 1 and "camera" No. 2 are enabled, shown by the projected rectangles.

These figures was made using the undocumented 3-D Studio screen print command. The procedure is described in Appendix I.

\subsection{Rendered Images}

The "camera" views were then rendered using 576 by 216 resolution with "aspect" set to 1.0. This corresponds to approximately $72 \mathrm{dpi}$ for the 16 bit grayscale image size as printed. The files were saved as 16 bit grayscale tif. For file resolutions higher than the display resolution the output needs to be set to "No Display" and the "Disk option" enabled. The tif files were imported into the Macintosh environment using the Apple File Exchange program and inserted into the WORD document.

Fig. 4 shows a view with the "camera" located on the machine axis viewing the outer limiter. Remember that the inner first wall will cast the proper shadows but is transparent to this view, only spotlight No. 2 is on. For the following discussion it will be assumed the lights are the IR camera and the projected pattern will be considered the camera view, as in an actual experiment. The angle of the outboard divertor clips the image and so there is no view 
directly below the camera. Furthermore, the vertical inner first wall and inner divertor prevent the view of the outer limiter 90 degrees away. This poses a serious problem and the only way to obtain complete toroidal coverage is by installing cameras at $45^{\circ}$ toroidal intervals. The alternative camera location on the inner wall does not have a view of the inner baffle and has a very oblique view of a very limited toroidal range, as shown in Figs. 20 and 21. Additionally this location presents serious access and maintenance problems.

The areas lit by the checkerboard pattern represent areas visible to an IR camera located at the position of the projector light. The size of the individual checkerboard squares represents the relative resolution and the aspect ratio of each individual square represents the obliqueness of the view. Graphite becomes reflective in the $\mathbb{R}$ at incident angles greater than about $70^{\circ}$. This corresponds to an aspect ratio of about 3 to 1 and for aspect ratios larger than this the temperature measurement will be suspect. As an example of an estimate of resolution taken from these general views, we will assume a camera with a 512 by 512 pixel detector. Then each checkerboard square (32 on a side) corresponds to 16 pixels. For the view of the outer divertor high heat flux region (Fig. 4), the long dimension of the checkerboard image projects to about 4 to $8 \mathrm{in}$. and the corresponding resolution would be in the range of about $1 / 2$ to 1 in. outside.

Fig. 15 shows the corresponding view of the inner baffle, viewed from

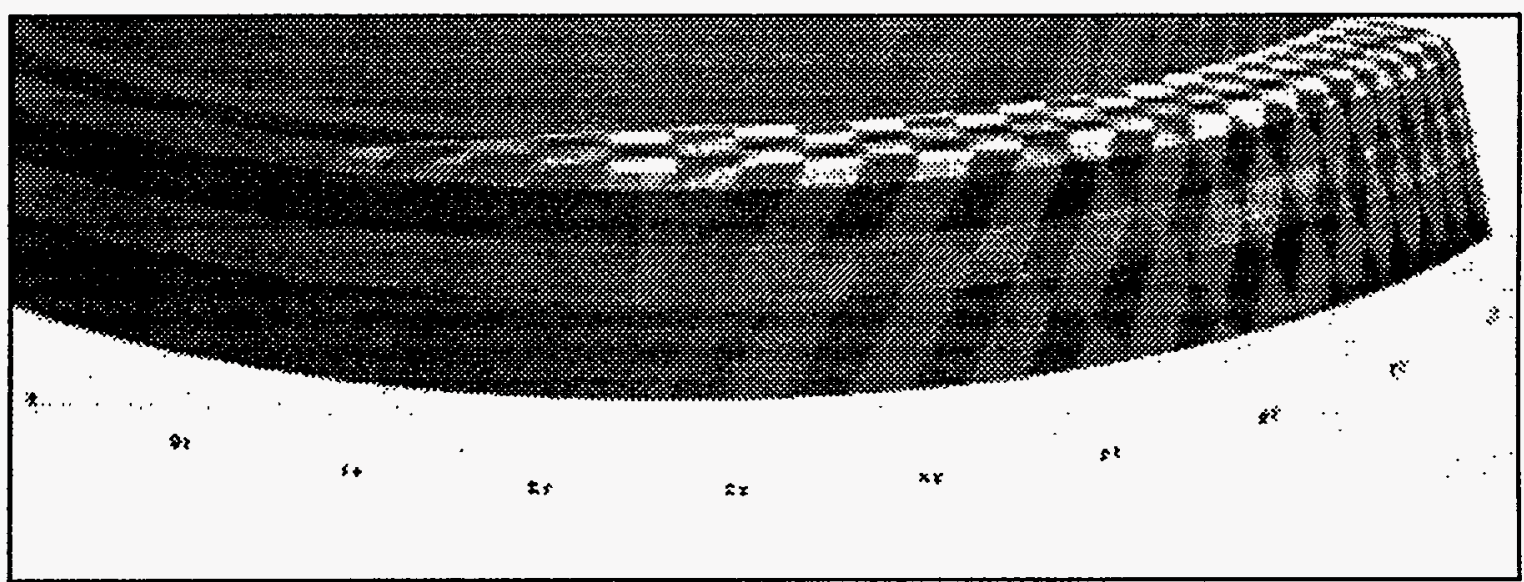

Fig. 15. View of inner baffle and divertor with spotlight No. 2 turned on.

For TPX currently assigned access is located at the $90^{\circ}$ and $180^{\circ}$ toroidal locations. In order to give some idea of the view possible with cameras located $90^{\circ}$ toroidally apart, Fig. 16 and Fig. 17 show the inner and outer views, similar to Fig. 3 and Fig. 15, except with spotlights To. 2 and No. 4 enabled. Both the coverage and resolution are reasonable, except for the view directly below the camera. As mentioned previously, for full coverage cameras are required at $45^{\circ}$ toroidal intervals. 


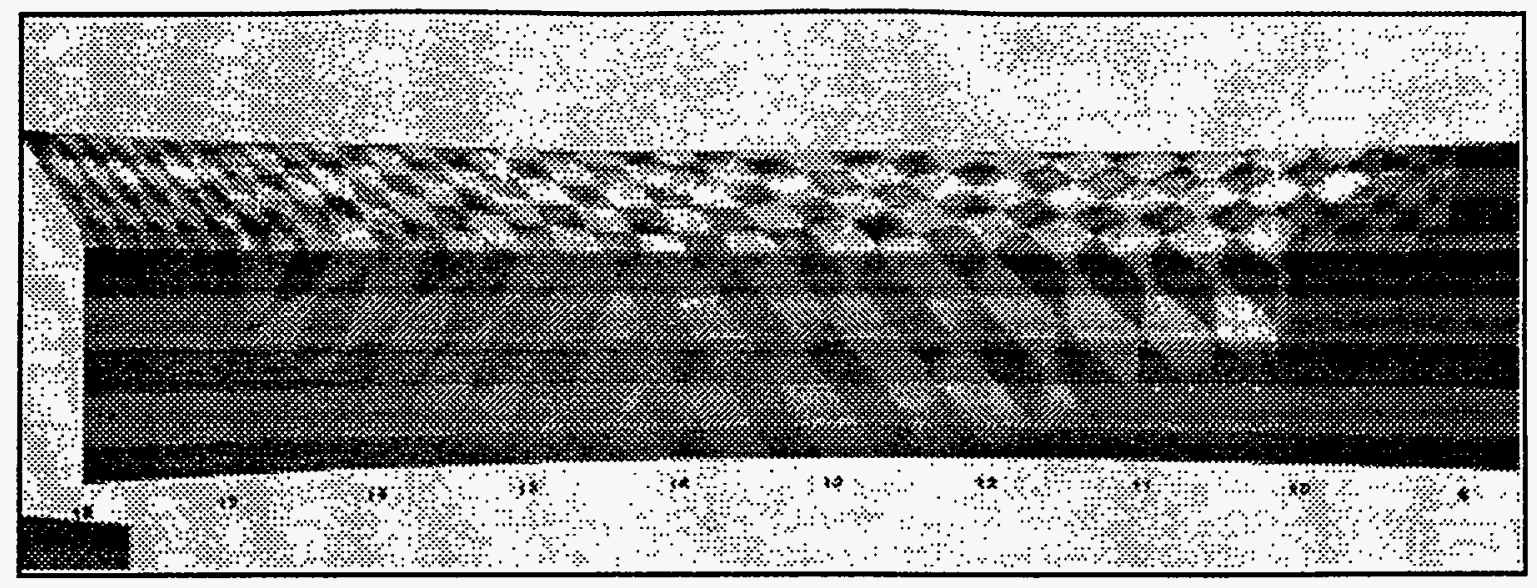

Fig. 16. View of outer divertor with spotlights No. 2 and No. 4 turned on.

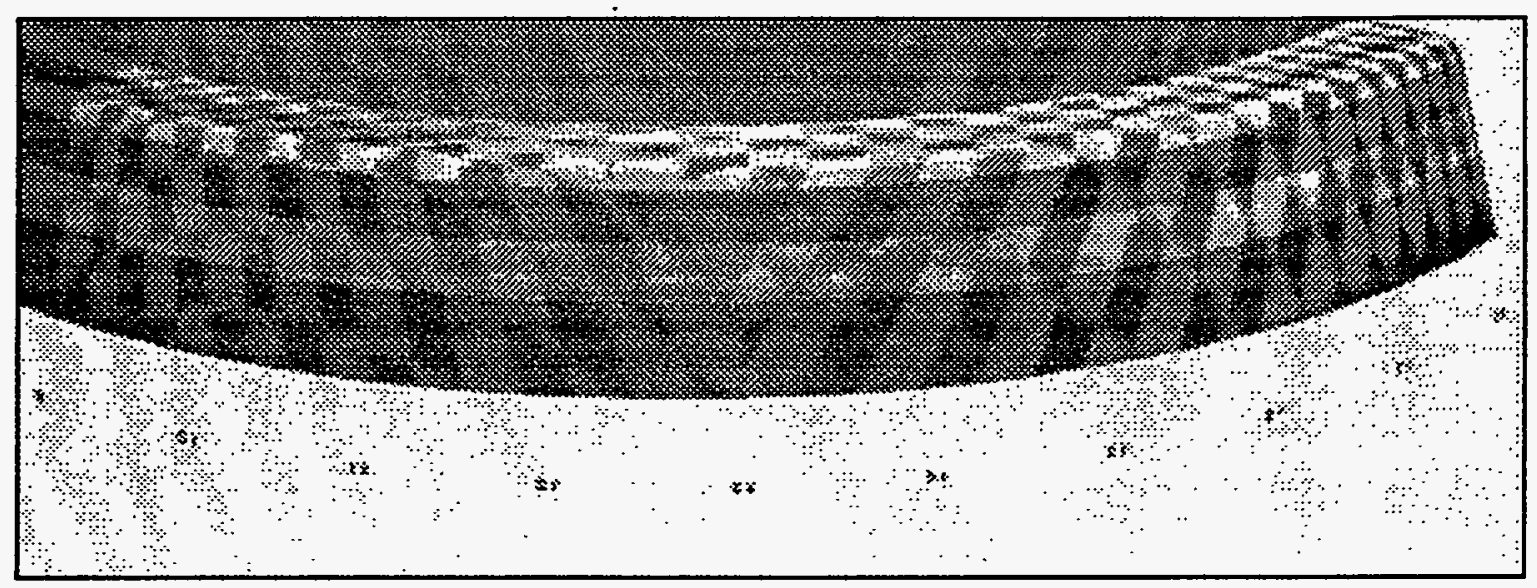

Fig. 17. View of inner baffle with spotlights No. 2 and No. 4 turned on.

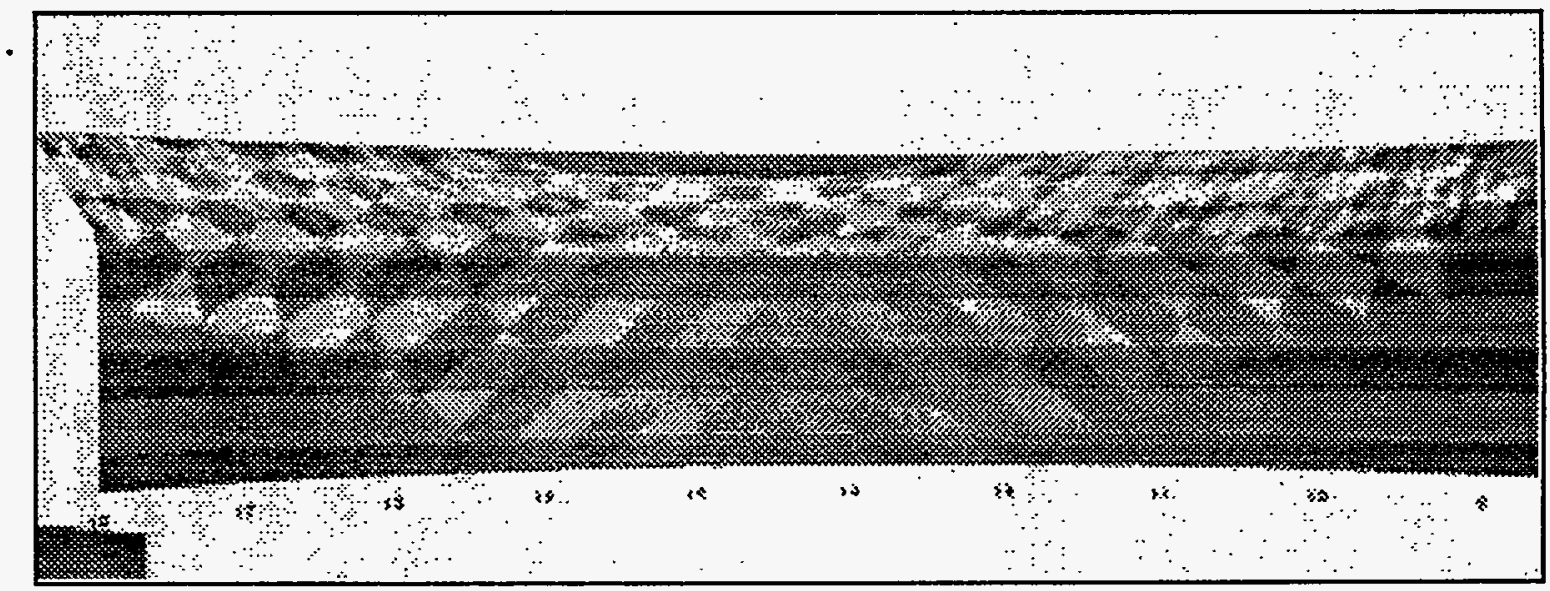

Fig. 18. View of outer divertor with spotlights No. 3 and No. 5 turned on.

Fig. 18 and Fig. 19 show the corresponding images for spotlights No. 3 and No. 5. In general the coverage and image quality is similar to that for spotlights No. 2 and No.4. This is the preferred camera location, radially 
inserted and remotely maintainable, viewing through a penetration in the passive stabilizer structure.

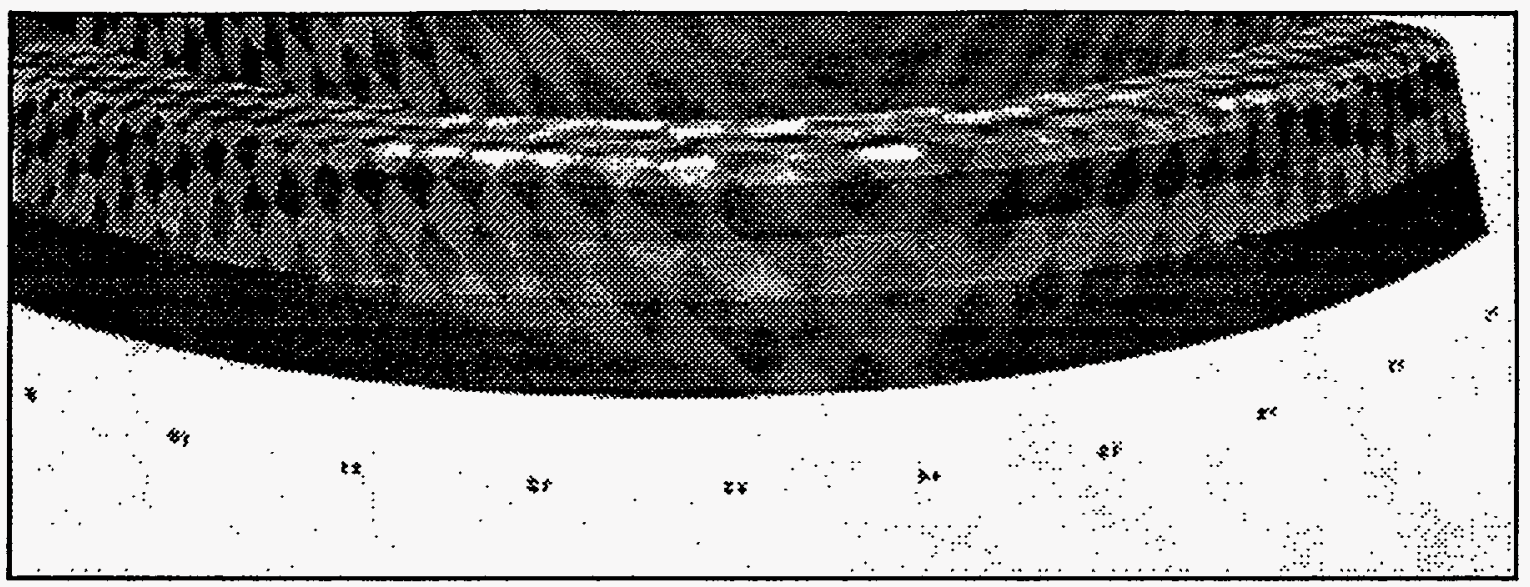

Fig. 19. View of inner baffle and inner divertor with spotlights No. 3 and No. 5 turned on.

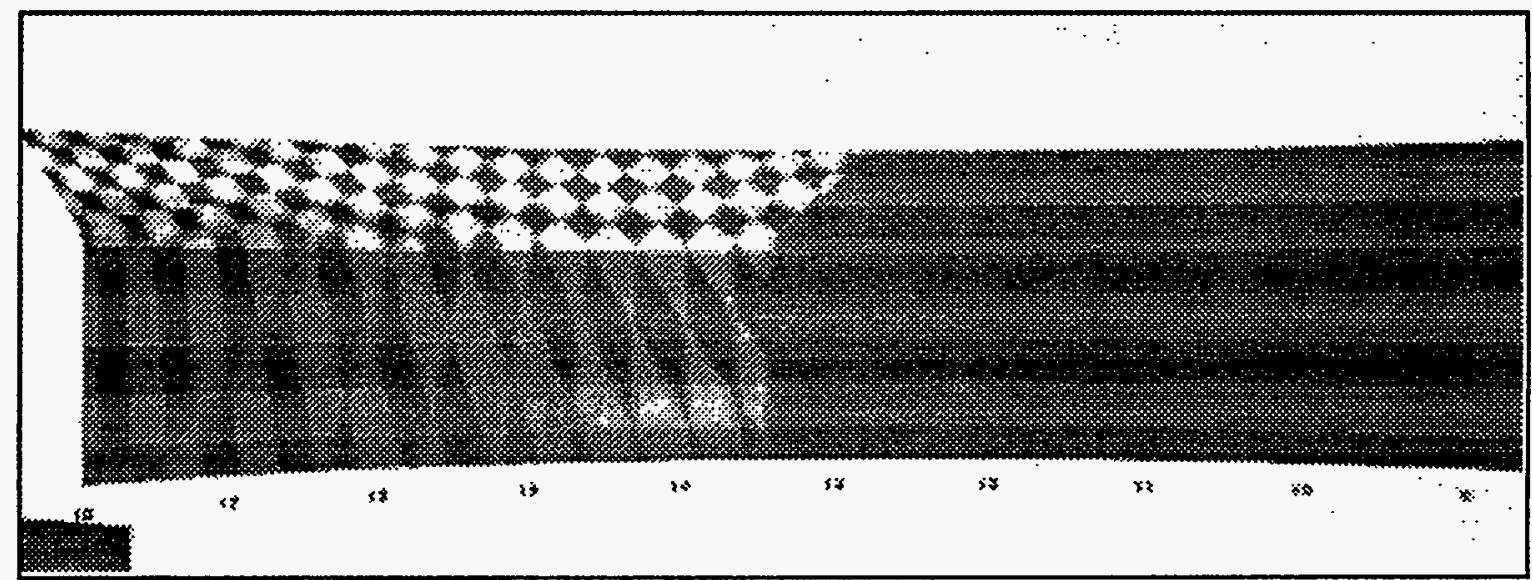

Fig 20. View of outer divertor with spotlight No. 1 on.

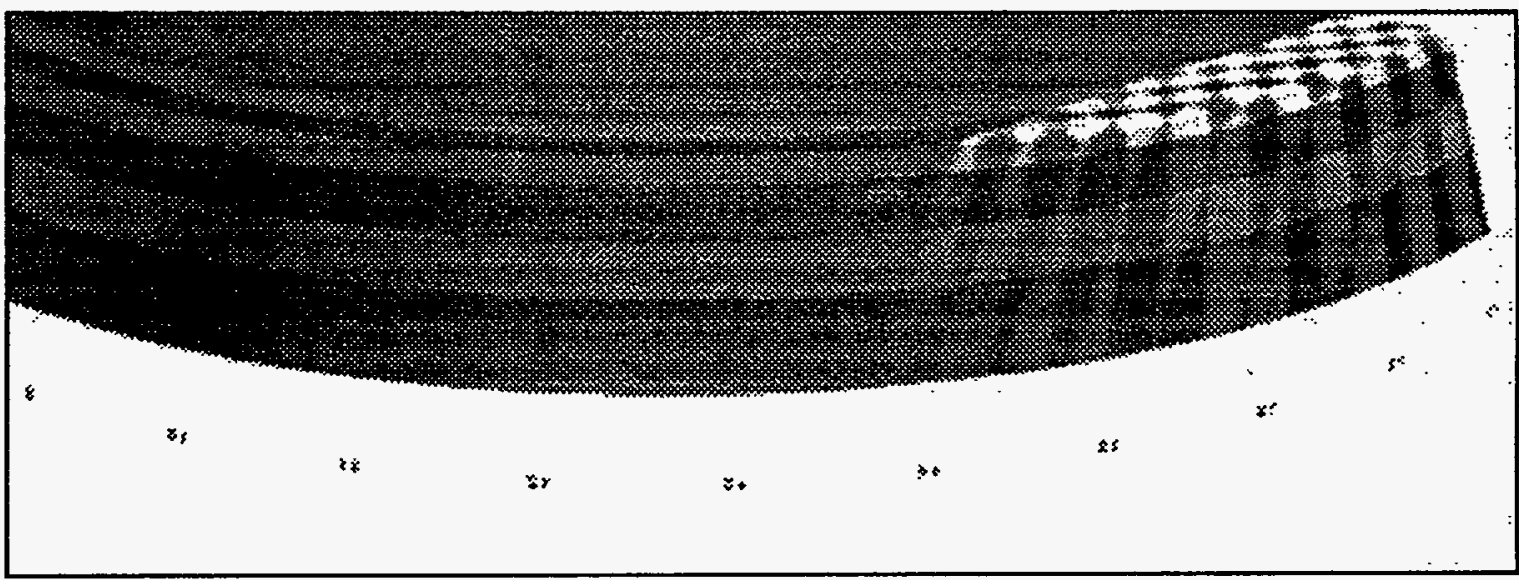

Fig. 21. View of inner baffle and inner divertor with spotlight No. 1 on. 
And for completeness, Fig. 20 and Fig. 21 show the views with spotlight No. 1. Only a limited toroidal range of the inner baffle is visible from this location with poor resolution. The toroidal view is very restricted because of clipping by the inner baffle and inner first wall cylinder.

\subsection{Analysis}

In order to generate quantitative values for the expected image resolution and incidence angles, a panoramic view was constructed. A projector image was generated by "creating" two circles in 2-D Shaper with radii of 9 and 11 units. A 64 on a side bitmap of this object was then constructed, as described in Sect. 5.4

For the views of the outer divertor the camera was located at the machine axis, the camera target at a radius of $60 \mathrm{in}$. and at an angle of $270^{\circ}$ and the field of view (FOV) set at $20^{\circ}$. A 36-sided polygon was created in the 2-D Shaper module with "angle" set to $270^{\circ}$ and modified so that only the bottom right quadrant existed, using "segment-delete" and "vertex-weld". In the Keyframer module a "path" was then assigned to the "camera" target using the "path-get-shaper" command. A set of rendered image files was then generated with the "Every Nth Frame" set to 6, so as to generate files every $20^{\circ}$. The images were saved at a resolution of 360 by 360 and transferred to the Mac using Apple File Exchange. A new file was opened in the application GraphicConvertor with a size of 1800 by 360 and the individual frames copied and pasted to construct the final panoramic view. Mr. Lemke, the author of GraphicConverter, was kind enough to write a beta version of his program to support keyboard entry of the "paste" coordinates, "paste at location", which greatly facilitated this operation. The panoramic view is shown in Fig. 5.

In order to calculate the resolution and the viewing angle, the coordinates of the ends of each axis of the projected ellipse were hand digitized from the GraphicConvertor display and entered into an EXCEL spreadsheet. The coordinates of each pattern center, the length of each axis and the resolution and normal incidence angle for each pattern were then calculated. The coordinates of each pattern center were included in a scale AutoCad drawing of the panoramic image and the resolution and incidence angle entered at each coordinate point. Then $a$, by eye, best fit set of contour plots were drawn. These are shown in Fig. 6 for the resolution and in Fig. 7 for the incidence angle.

For the inner divertor view the situation is considerably more complicated. Both the camera and the camera target have to be panned and two "path" polygons are required. For the example shown here the step size was set at $5^{\circ}$. Because of the camera view divergence, the reconstruction has to be made from many small strips with a long focal length for the "camera". The camera was located on the machine axis with an elevation of $-55^{\prime \prime}$. The target radius was set at $80^{\prime \prime}$, the outer most radius of the inner baffle, and the FOV at $5^{\circ}$, and both the camera and camera target located at $270^{\circ}$. Using the "camera-perspective" command, this command maintains a constant view as the lens focal length is changed, the camera was moved to $500^{\prime \prime}$ at $270^{\circ}$. "Snap" was enabled for precision location. Two Keyframer "paths" were constructed in 2-D Shaper, using 72 segment polygons trimmed as described above, with radii of 80 " and 
500" and attached to the camera and camera target. The camera view was then rendered in Keyframer with "every Nth" enabled. Resolution was set to 64 by 216 pixels. The picture strips were pasted together in GraphicConvertor to produce an assembled image. The effect of finite step size and camera divergence can be seen in the final image. This image was scaled nonproportionally by the slope of the baffle $\left(72.6^{\circ}\right)$ in order to correct for the distortion produced by the coned shape of the inner baffle. The final image is shown in Fig. 22.

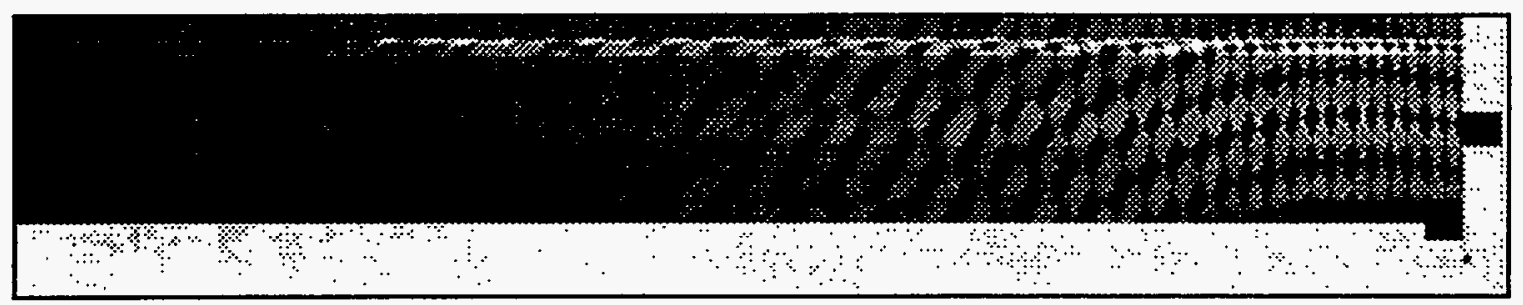

Fig. 22. Panoramic view of inner baffle assembled from $5^{\circ}$ strips and stretched vertically by $4.4 \%$ to compensate for baffle cone shape.

\section{Conclusions}

The procedures described here provide a fast and flexible technique for developing detailed images of 3-D camera views of the tokamak interior. From these images, reasonably accurate estimates of image resolution and viewing angles of incidence may be obtained. The corresponding experimental problem of interpreting the camera views can similarly be solved by this technique. The camera image (probably a video frame) will be converted to a bit map and installed in the spotlight projector. The spotlight parameters will be adjusted until the projected image registers with the divertor structure. The divertor structure, with the projected image can then be viewed from any convenient position and the resulting image rendered and displayed.

An analytic solution was developed for interpreting views of more complex surfaces and for higher precision measurements. The results of this calculation were compared with one set of results from the imaging technique and were in good agreement.

The combination of the 3-D view generated by these procedures and the analytical resolution and viewing incidence angle obtained from the analytic calculation provide a complete solution to the viewing diagnostic design requirement.

The obtainable coverage, resolution and angles of incidence for the presently assigned IR camera locations on TPX are optimistically marginal. If cameras are located at toroidal intervals of $45^{\circ}$, full coverage is possible with somewhat improved viewing performance. 


\section{Acknowledgments}

This work was performed for the TPX project and was supported by the U.S. Department of Energy under contract No. DE-AC02-76CH03073.

The authors would like to thank Tom Brown and Pat Murray, TPX project, Emilia Solano and Bill Craven, University of Texas Fusion Research Center and Thorsten Lemke, author of GraphicConverter for their help in this study. The assistance Z. Simpson and B. Garland, Austin, Texas in applying the 3-D Studio software in this study is gratefully acknowledged.

\section{Appendix I 3-D Studio Print-Screen Command}

To enable this command, before entering 3-D Studio set the environment variable "set screengrab= yes". This may be included in the autoexec.bat file. Then the screen image can be saved with the command "control printscreen".

Remember to move the cursor either out of the way or wherever is required to emphasize the image.

. The cursor will lockup for several seconds (or longer) after which the display will ask for a file name for the screen dump file. The file will be written in the format used for the extension, i.e., dump.tif will produce a tif file. The screen views were converted to black and white line art using the HiJaak application before saving as a monochrome tif file. Because of the large number of color changes required, it is recommended that the "color-list color changes" "clear" command be executed after every successful color change. The tif files were then saved as 16 bit monochrome files and imported into the MAC shareware application GraphicConvertor via Apple File Exchange. The foreground/background colors were corrected for the normal HiJaak aberrations. The image was scaled using "copy as" to the required size for the final WORD document ( 6 " at $150 \mathrm{dpi}$ is 900 pixels) and then converted into a new drawing with "New Picture with Clipboard". The captions were added and the drawing copied directly to the WORD document.

Appendix II Program for divertor resolution calculation

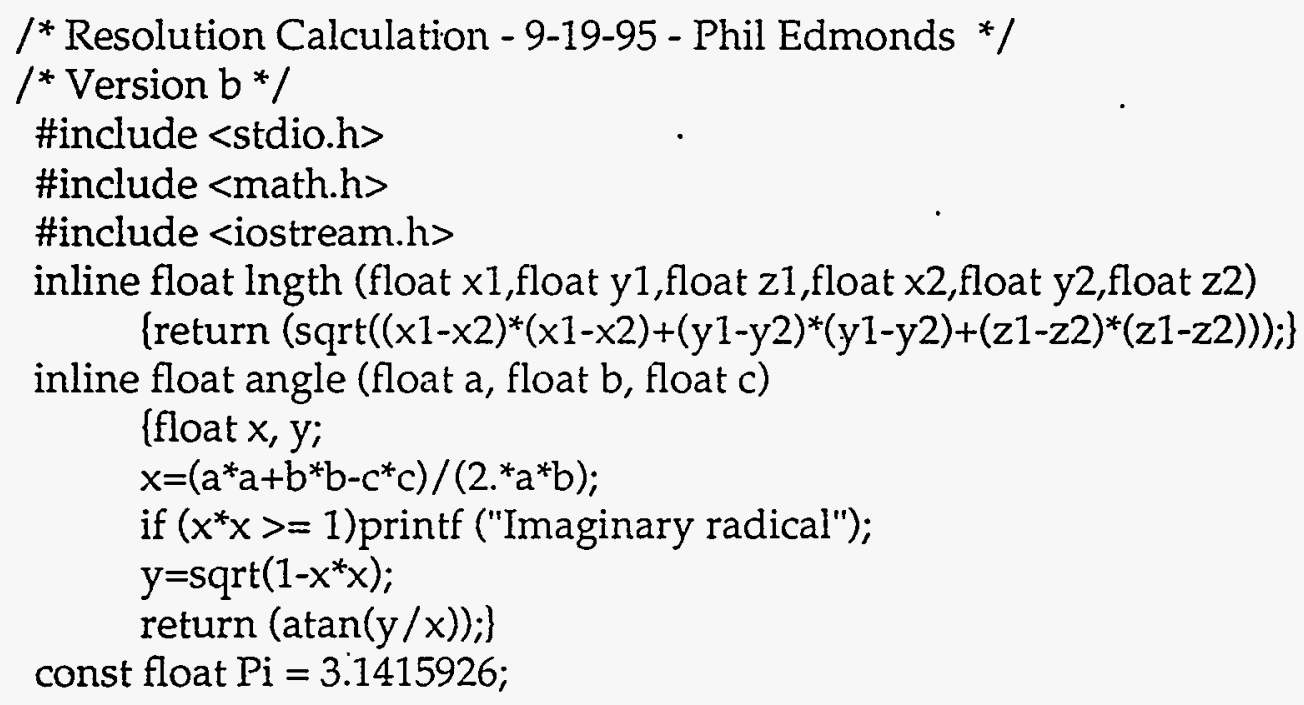


float zstep, tstep,rtod, dtor, limage,lpix, $x n, y n, z n, t n$, lcsurf, theta, phi,res; float $\mathrm{rcyl}=80$., flens $=10$, npix $=512$;

float fov $=21.3605$;

$1 *$ half full angle from 3-D Studio FOVTST *

float $x c=89 ., y c=0 ., z c=39$;

$1 *$ Camera lens coordinates *

float $\mathrm{xt}=66.4, \mathrm{yt}=-41.6, \mathrm{zt}=-40.2$;

float zstart $=-68$, zend $=-40$;

float tstart $=150-180$, tend $=100-180$;

main()

\{

FILE *fp;

fp=fopen("resout.dat", "w");

cout $<<" \backslash n$ Enter step size (inches): ";

cin > zstep;

rtod $=180 . / \mathrm{Pi}$;

dtor $=\mathrm{Pi} / 180$;

limage $=2 .{ }^{*}$ flens ${ }^{*}$ atan (fov ${ }^{*}$ dtor);

lpix=limage/npix;

$1 *$ length of image plane * 1

tstart $=$ tstart ${ }^{*}$ dtor;

$1 *$ length of pixel */

tend $=$ tend ${ }^{*}$ dtor;

tstep $=$ zstep $/$ rcyl;

fprintf(fp," zn tn res theta phi");

zn=zstart;

while $(\mathrm{zn}<=$ zend) \{

tn $=$ tstart;

while $(\mathrm{tn}>=$ tend $)$ (

$\mathrm{xn}=\mathrm{rcyl}^{*} \cos (\mathrm{tn}) ; \mathrm{yn}=\mathrm{rcyl}{ }^{*} \sin (\mathrm{tn}) ; \quad I^{*}$ divertor surface coordinates $* /$

lcsurf $=\operatorname{lngth}(x c, y c, z c, x n, y n, z n) ; \quad / *$ camera to surface distance */

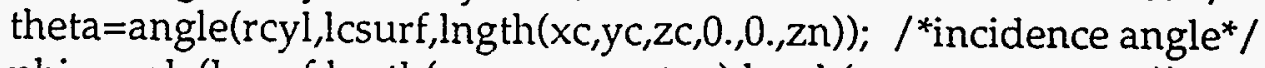

phi=angle(Icsurf, lngth $(x c, y c, z c, x t, y t, z t), \operatorname{lngth}(x n, y n, z n, x t, y t, z t))$; res $=$ lpix ${ }^{*} \operatorname{lcsurf} /\left(\right.$ flens ${ }^{*} \cos ($ theta $\left.)\right) ; I^{*}$ resolution in inches/pixel * fprintf(fp,"\n\%7.3f \%7.3f \%7.3f \%7.3f \%7.3f"

,zn,-(180+tn*rtod),res, theta*rtod,phi*rtod);

tn=tn-tstep;

)

zn=zn+zstep;

)

fclose(fp);

\}

\section{DISCLAIMER}

This report was prepared as an account of work sponsored by an agency of the United States Government. Neither the United States Government nor any agency thereof, nor any of their employees, makes any warranty, express or implied, or assumes any legal liability or responsibility for the accuracy, completeness, or usefulness of any information, apparatus, product, or process disclosed, or represents that its use would not infringe privately owned rights. Reference herein to any specific commercial product, process, or service by trade name, trademark, ence herein to any specific comes not necessarily constitute or imply its endorsement, recommanufacturer, or otherwise does not necessarily constituent or any agency thereof. The views mendation, or favoring by the United States of and opinions of authors expressed herein do not 
